\title{
SOBRE ENCLISIS Y ELISIÓN EN GRIEGO ANTIGUO
}

\author{
About enclisis and elision in ancient greek
}

Rodrigo Him Fábrega*

\begin{abstract}
RESUMEN
El proceso de elisión del griego antiguo se analiza como un recurso de restructuración de los constituyentes prosódicos, que se activa en el ámbito de la fonología oracional después de las reglas que definen los patrones de acentuación. Así concebida, la elisión trae apoyo independiente para la tesis de que la enclisis es un mecanismo de adjunción encarrilado al acomodo de expresiones desprovistas de ligamen métrico.

Palabras clave: Griego antiguo, elisión, enclisis, acento grave, fonología métrica.
\end{abstract}

\begin{abstract}
The Ancient Greek process of elision is analyzed as a kind of restructuring of prosodic constituents, which occurs in sentence phonology after the rules that define the patterns of accentuation. With such approach, the elision brings independent support to the idea that enclitic formation is a mechanism of adjunction aimed to accommodate metrically disconnected expressions.
\end{abstract}

Key Words: Ancient Greek, elision, enclisis, grave accent, metrical phonology.

Universidad de Panamá. Panamá.

Correo electrónico: bundiorody@gmail.com

Recepción: 6/6/2014 Aceptación: 10/4/2015. 


\section{Preámbulo}

Este estudio escudriña el dominio de las relaciones que conectan el sistema prosódico con la regla de elisión del griego antiguo. Le adjudica a la elisión el carácter de un proceso de reajuste silábico que ocurre con posterioridad a la fase en que los enclíticos se agrupan en torno a algún término ortotónico, pero antes de que la proclisis reacomode la secuencia de los constituyentes en la esfera de la fonología oracional. Enclisis y proclisis se caracterizan como mecanismos postléxicos de formación de palabras compuestas, cuyo propósito es asegurar la plena interpretación prosódica de los constituyentes. ${ }^{1}$

\section{Propiedades de la elisión}

La elisión es un fenómeno de juntura (Allen 1973:226). Se produce cuando dos vocales de palabras distintas entran en contacto en la cadena de la frase. En tal entorno, se suprime la última vocal de una palabra seguida por otra que comienza con vocal o espíritu áspero (aspiración). El fenómeno se da en casos como los de (1).

(1)

\begin{tabular}{|c|c|}
\hline 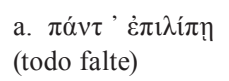 & (Plat. Rep. 573e) \\
\hline $\begin{array}{l}\text { b. "̌́t ' } \alpha i \sigma \chi i \omega v \\
\text { aun más fea) }\end{array}$ & (Aristoph. Eccl. 1078) \\
\hline 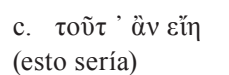 & (Plat. Rep. 402d) \\
\hline
\end{tabular}

El sitio de la elisión se señala en la escritura griega mediante el apóstrofo. La forma plena de los términos apocopados de los ejemplos

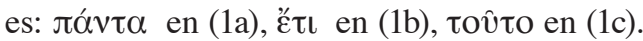

Cuando la segunda palabra de la secuencia se inicia con aspiración, esta se alinea con la consonante que subsista al final de la primera palabra, si dicha consonante es una oclusiva sorda, como en (2a-b). Con segmentos distintos, como la fricativa de (2c), debe suponerse que la aspiración desaparece (Allen 1973:229).

$$
\begin{aligned}
& \text { (2) } \quad \text { av́ } \chi \theta^{\prime} \quad \check{\lambda} \eta v \\
& \text { (la noche entera) }
\end{aligned}
$$

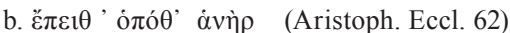 \\ (luego, cuando el hombre)

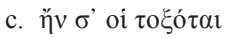 \\ (Aristoph. Eccl. 258) \\ (si a ti los arqueros)
}

Donde las formas plenas correspondientes

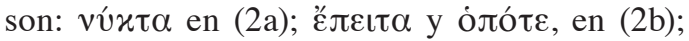

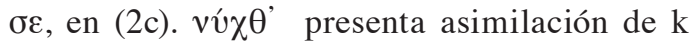
tras la elisión.

\subsection{Dominio de la elisión}

No es cualquier vocal la que se elide. La supresión afecta solo a las vocales breves inacentuadas, con excepción de $v$ ([y]). Se puede postular, en consecuencia, para la elisión, la regla que se formaliza en (3), para la que $V_{i}$ es una vocal breve átona distinta de $v$ y $v$ y $\langle$,$\rangle señalan$ los lindes de palabra fonológica.

$$
\begin{aligned}
& \text { Regla de elisión: } \\
& \left.\mathrm{V}_{\mathrm{i}} \rightarrow \varnothing /_{----}(-1)\right\rangle\left\langle(\mathrm{h}) \mathrm{V}_{\mathrm{j}}\right.
\end{aligned}
$$

Sin salir de los confines del repertorio estándar de rasgos fonológicos (Chomsky y Halle 1968), la regla (3) debe entenderse como resumen de dos procesos distintos que se aplican en el mismo entorno condicionante. ${ }^{2}$ El símbolo $\dashv$ se introduce optativamente a fin de incluir en la regla el caso en que la vocal suprimida es un segmento de un enclítico (cf. representación (10), abajo).

\subsection{Facultatividad de la elisión}

Datos como los que aparecen en (4),

\begin{tabular}{|c|c|c|}
\hline \multirow[t]{5}{*}{ (4) } & 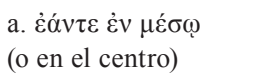 & (Plat. Rep. 523d) \\
\hline & 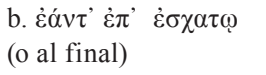 & (Plat. Rep. 523d) \\
\hline & 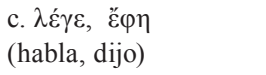 & (Plat. Rep. 445d) \\
\hline & $\begin{array}{l}\text { d. } \lambda \varepsilon^{\prime} \gamma^{\prime}, \check{\varepsilon} \varphi \eta \\
\text { (habla, dijo) }\end{array}$ & (Plat. Rep. 414c) \\
\hline & $\begin{array}{l}\text { e. } \mu \eta ́ \tau \varepsilon \dot{\alpha} \delta ı \kappa \varepsilon \tau v \\
\text { (ni cometer injusticia) }\end{array}$ & (Plat. Rep. 359b) \\
\hline
\end{tabular}
donde alternan formas plenas y apocopadas en contextos idénticos o semejantes, prueban que la elisión opera de manera facultativa. 
f. $\mu \tau^{\prime} \tau^{\prime} \dot{\alpha} \delta 1 \kappa \varepsilon \tilde{i} v \quad$ (Plat. Rep. 359a) (ni cometer injusticia)

La ruptura discursiva de (4d), señalada por la coma, no impide la elisión, lo cual sugiere que las palabras en este caso podían ser pronunciadas como una secuencia prosódica continua.

\subsection{Elisión y acento grave}

El acento grave es un rasgo de las expresiones oxítonas que no se hallan al final de una unidad de entonación. Dicho con sentido amplio, tales expresiones sufren la remoción del acento agudo si se insertan en una posición que no vaya seguida por una pausa, como lo muestra la alternancia de (5).

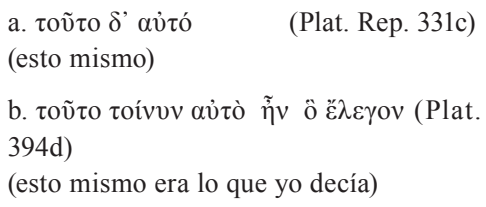

La remoción adopta la pauta de la regla (6), para la que $X$ vale por un término ortotónico o un proclítico, y tiene efectos sobre todas las palabras oxítonas ${ }^{3}$ (excepto, el interrogativo $\tau i ́ \zeta$, 'quién').

$$
\begin{gathered}
\text { Regla de remoción de acento agudo: } \\
\text { +Agudo }
\end{gathered}
$$

Los datos de (7) indican, por otra parte, que la vocal marcada con acento grave puede ser objeto de elisión.

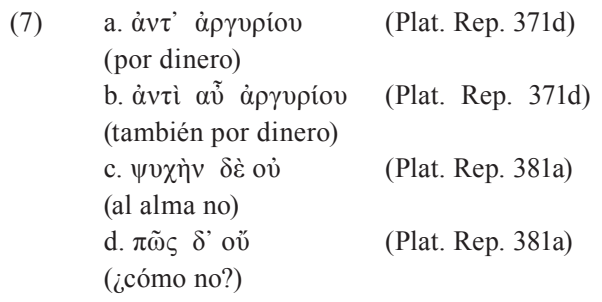

Este fenómeno entraña que las vocales que se marcan con el rasgo del acento grave deben ser consideradas propiamente como vocales inacentuadas, algo que ya fue observado por Trubetzkoy (1939:227). Se trata, así, de la ausencia o la privación del acento (Vendryes 1938:35).

Pero dicha privación no supone la carencia absoluta de propiedades prosódicas. El acento grave surge de un proceso que se localiza en la fonología de la oración, y debe distinguirse de la ausencia irrestricta de acento. Como dice Vendryes (1938:41), la sílaba aguda que cambia a grave difiere psicológicamente de la átona común. En la aproximación que aquí se elabora, la diferencia puede expresarse en el plano de la estructura métrica. Así, la existencia en cualquier estadio de derivación de un acento agudo implica que la expresión que lo aloja ha sido analizada métricamente. La marca de acento grave entonces se convierte en el signo de la estructura métrica existente, a despecho de que la sílaba que la soporta parezca fonéticamente indistinta de una sílaba átona. La diferencia psicológica a que alude el comentario de Vendryes se sigue de que la vocal con acento grave se interpreta como núcleo de un constituyente métrico subyacente (adormilado, en la terminología de la tradición). Esto, en principio, provee una explicación para el hecho de que cuando dicho núcleo es sometido a elisión el aparato de análisis prosódico proceda a recomponer el esquema métrico (el pie de nivel de palabra, en el sentido de Steriade (1988:276)), adjudicando la condición de núcleo a la última vocal subsistente del ítem. Tal fenómeno de restructuración ha sido observado en otras lenguas (cf. Kenstowicz 1996:259s.). La ilustración se da en (8).

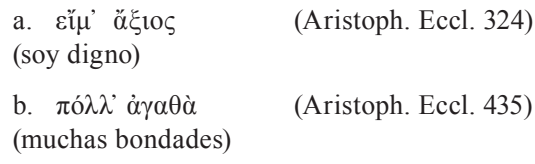

(Aristoph. Eccl. 324)

(Aristoph. Eccl. 435)

Donde las formas plenas de las palabras apocopadas son las oxítonas sỉuí, en (8a), y $\pi \mathrm{o} \lambda \lambda \dot{\alpha}$, en (8b).

La posibilidad de supresiones de la clase de las de (8) muestra que la elisión no considera la diferencia métrica que distingue a la sílaba con acento grave de la sílaba átona común. El proceso se atiene a las características fónicas 
superficiales, para las que la una y la otra son meramente sílabas privadas de tono agudo.

\section{Enclisis}

La regla de remoción (6) no se aplica cuando el término oxítono está delante de un enclítico. Aunque en este entorno no existe pausa, el rasgo de acento agudo sobre la última vocal de la expresión que antecede al enclítico es obligatorio. La ilustración se da en (9).

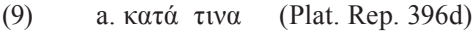 \\ (con alguno)

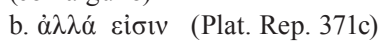 \\ (pero son)}

La aparente excepcionalidad se explica admitiendo la idea de que los enclíticos se adjuntan a la expresión (ortotónica) que les precede con objeto de obtener visibilidad en el plano de la prosodia. Por ejemplo, (9a) se transforma mediante adjunción en (10), donde como antes los paréntesis angulares señalan los lindes de palabra fonológica, mientras que el signo $\dashv$ marca las fronteras del enclítico.

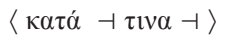

La adjunción ${ }^{4}$ del enclítico tiene la consecuencia de que la vocal acentuada se desplace desde el final hacia el interior de la palabra, por lo que viene a estar excluida del dominio de aplicación de (6). Un resultado suplementario del análisis es que sobre (9) no tenga ningún efecto la regla de elisión.

La enclisis, según lo expuesto, entra en actividad con antelación a las reglas de remoción y de elisión. La remoción expande el dominio de la elisión, mientras que la enclisis restringe el dominio de la remoción. Por lo tanto, el orden de los procesos debe ser: (i) enclisis, (ii) remoción, (iii) elisión.

\subsection{Composición post-léxica}

La representación de (10) concibe la secuencia de preposición más enclítico como una suerte de palabra fonológica compuesta. En los compuestos léxicos del tipo de $\mu \varepsilon \gamma \alpha \lambda$ o $\varrho \varepsilon ́ \pi \varepsilon\llcorner\alpha$ 'nobleza' se observa la tendencia a retrotraer el acento hasta donde lo permita la regla de limitación (Vendryes 1938:188ss.). La forma de (10) se adecua a esta pauta. ${ }^{5}$

Considérese ahora expresiones de la clase de (11), en las que el elemento que hospeda al enclítico está doblemente acentuado.

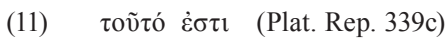
(esto es)

Este fenómeno se caracteriza propiamente haciendo que la enclisis imponga una marca de acento agudo sobre la última vocal del elemento huésped siempre que la misma está disponible para recibirla. La disponibilidad se sigue, como en el caso de (10), del carácter extramétrico de la sílaba final de la expresión ortotónica (cf. Steriade 1988:273). En los términos de la aproximación de Allen (1973:234), dicha sílaba no está incluida en la contonación, y puede servir así como unidad de un constituyente prosódico subsidiario. ${ }^{6}$ Un recurso de tal carácter es, en efecto, indispensable para acomodar la secuencia de sílabas del compuesto al esquema de limitación de la prosodia griega, que exige una marca de acento en alguna de las tres sílabas finales de la palabra. La existencia de dos contonaciones es consistente con el hecho de que (11) no es un compuesto léxico, sino que se construye en la zona post-léxica de la fonología, análogamente a como surgen por contracción compuestos post-léxicos de la clase de los de (12).

(12) a. $\mu \varepsilon ́ v \tau \alpha \ddot{v} v$ (Aristoph. Eccl. 650)

(en caso tal, sin duda)

b. $\mu \varepsilon ́ \vee \tau о \mho ̋ \varphi \alpha \sigma \kappa \varepsilon \nu$ (Aristoph. Eccl. 410)

(dijo efectivamente)

La composición produce para (11) la representación de (13).

(13) 〈

De tal representación se desprende que el acento invocado por la enclisis está exento tanto de remoción, como de elisión.? 


\subsection{Enclisis adquirida}

En griego, deben distinguirse los enclíticos inherentes de los condicionados. Los primeros solo reciben tono agudo cuando preceden a otro enclítico, en el fenómeno de la sinenclisis (Vendryes 1938:87s., Allen 1973:244). Los segundos son términos portadores de acento léxico que adquieren la enclisis por condicionamiento sintáctico. Enclítico inherente es, por ejemplo el pronombre $\mu \mathrm{ol}$ (dativo singular de primera persona), mientras que $\phi \eta \mu$ ' 'digo' es un enclítico condicionado.

Los enclíticos condicionados se encuentran en alternancia con formas ortotónicas que se insertan en los contextos que no producen enclisis. Esto ocurre, en especial, en comienzo de frase, $y$ en situaciones de lectura enfática (Vendryes 1938:108, 238s.). La alternancia se observa en los siguientes ejemplos:

\begin{tabular}{|c|c|c|}
\hline (14) & 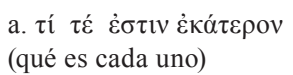 & (Plat Rep. 368c) \\
\hline & 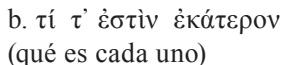 & (Plat. Rep. \\
\hline
\end{tabular}

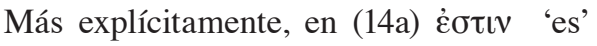
exhibe enclisis condicionada, mientras que en (14b) se mantiene como expresión ortotónica, por hallarse en el caso de la lectura enfática. ${ }^{8}$ En consecuencia, en (14a), mediante adjunción consecutiva de los dos enclíticos, la vocal de la partícula $\tau \varepsilon$ (un enclítico inherente) se ve protegida de la elisión. Pero en (14b), donde queda al final de la palabra compuesta, sufre apócope. Los procesos de derivación de dan en (15), para (14a), y en (16), para (14b).

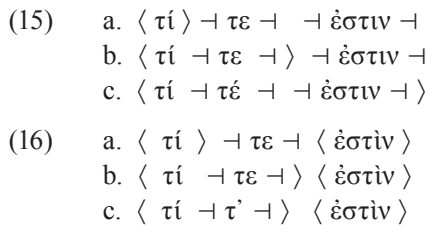

\section{Resilabificación}

La elisión presenta el cuadro de atributos de los procesos que pueden reacomodar la estructura prosódica previamente construida. Exceptuando al grupo de palabras oxítonas ilustrado arriba con los ejemplos de (8), el cual, sometido a la regla de remoción, y luego a la de elisión, pierde el núcleo de la estructura prosódica, el reajuste no llega a eliminar los constituyentes métricos existentes, pues los acentos especificados, bien en la fase léxica, bien en la post-léxica, se preservan. Para comprobarlo, considérese (17).

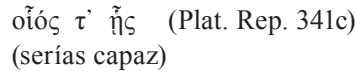

Según lo anterior, la forma original de la que procede (17) es la de (18).

$$
\langle\text { oĩos }\rangle \dashv \tau \dashv\langle\tilde{n} \varsigma\rangle
$$

La enclisis adjudica acento delimitador sobre la mora final del primer término de la expresión:

(19) 〈ớó $\dashv \tau \varepsilon \dashv\rangle\langle\tilde{n} \varsigma\rangle$

Luego, la elisión elimina la rima del enclítico:

(20) 〈õós $\left.\dashv \tau^{\prime} \dashv\right\rangle\langle\tilde{n} \varsigma\rangle$

La falta de núcleo hace que desaparezca la sílaba final del compuesto, por lo que $\tau$ queda desconectada de la estructura métrica. Entonces, la consonante se reacomoda como cabeza de la sílaba inicial de la palabra siguiente, según se representa en (21).

(21) $\langle$ ỡós $\rangle\langle\tau$ 'ก̃ $\rangle$

Interesa en este punto observar que en (21) se satisfacen las condiciones para la aplicación de la regla (6). Sin embargo, oíó en oí̀̀ร. Esto se debe a que la regla de remoción se activa antes de la elisión, de modo que en la etapa en que esta altera la secuencia de sílabas, aquella ya no tiene efecto.

La resilabificación de las secuencias de tipo VC.V como secuencias de tipo V.CV está ampliamente difundida en las lenguas (Steriade 1988:287, Harris 1993:181), y debe admitirse que este es el sentido en que se combinan los 
segmentos puestos en contacto por la elisión. Una consonante que queda descarrilada busca asociarse con una vocal que posee autonomía prosódica. El proceso altera mínimamente la estructura, porque la cabeza no interviene en el cálculo del peso o la cantidad de la sílaba. Y como ha notado Allen (1973:227), la consonante descarrillada no crea posición para la vocal que le antecede, lo cual prueba que no funciona como coda de su núcleo. Por ejemplo, en (22a) $\tau$ no es coda de ö, ni en (22b) $\sigma$ lo es de í.

\section{(22) a. ö $\tau^{\prime} \dot{\varepsilon} \sigma \kappa о \pi о v ́ \mu \varepsilon \theta \alpha \quad$ (Aristoph. Eccl. 193) \\ (Lo que veíamos)

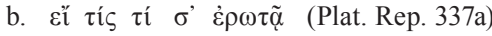 \\ (si alguien te preguntara algo)}

La oración (22a) procede de la estructura dada en (23), donde el símbolo $\ulcorner$ designa los lindes de un proclítico.

$$
\ulcorner\varepsilon \dot{\imath}\ulcorner\dashv \tau 1 \zeta \dashv \dashv \tau \dashv \dashv \sigma \varepsilon \dashv\langle\dot{\varepsilon} \rho \omega \tau \tilde{\alpha}\rangle
$$

La asignación de acento delimitador por parte del primer enclítico convierte a $\varepsilon \grave{i}$ (proclítico) en expresión prosódicamente autónoma. La elisión destraba la consonante del pronombre personal, que entonces se asocia con la palabra siguiente. El resultado se representa en (24).

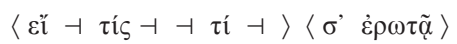

Los lindes de $\sigma \varepsilon$ se difuminan al perderse el constituyente que delimitaban, mientras que los lindes del proclítico eỉ se transforman en los de una expresión ortotónica (عí) ).

\section{Elisión y proclisis}

Un argumento a favor de la tesis de que la elisión ocasiona un reajuste de segmentos en el sentido VC.V hacia V.CV lo proporcionan las características de los proclíticos en ejemplos como los siguientes:

\footnotetext{
(25) a. $\dot{\alpha} \lambda \lambda \dot{\alpha}$ Eíoı (Plat. Rep. 371c)

(pero son)

b. $\dot{\alpha} \lambda \lambda$ ov่ $\kappa \varepsilon \chi \omega \rho ı \mu \mu \varepsilon ́ v o v$ (Plat. Rep. 524c)

(pero no separado)
}

$$
\begin{aligned}
& \text { c. } \mu \eta \delta^{\prime} \text { ö } \tau \text { (Plat. Rep. 337b) } \\
& \text { (ni que) }
\end{aligned}
$$

De acuerdo con lo observado atrás, el enclítico atrae acentuación oxítona en la palabra que lo hospeda en (25a), dada antes en (9b). La enclisis protege a la vocal acentuada de la elisión. En (25b) y (25c), en cambio, la vocal ha sido suprimida. Supóngase que en estas dos expresiones la vocal del segundo término se desplaza de su sílaba matriz para ocupar el sitio que la elisión dejó vacante, y no a la inversa, como se hizo arriba (esto es, que la consonante descarrilada satura el sitio vacío de la cabeza del constituyente que la sigue). Un acercamiento de tal carácter produciría resultados anómalos en varios respectos: (i) siendo la negación una palabra monovocálica, que regularmente se reviste de la figura del proclítico, se conduciría en (25b) como un enclítico; (ii) la negación parece sufrir enclisis, pero no induce acentuación oxítona en el nexo $\dot{\alpha} \lambda \lambda \alpha ́$, ni protege la vocal final del mismo de la elisión; (iii) la expresión que resulta de la resilabificación carece de visibilidad prosódica, ya que no cuenta con un núcleo acentuado; (iv) el núcleo ov̉ es una vocal larga ( [u:] ), mientras que la posición que tendría que llenar en $\dot{\alpha} \lambda \lambda \dot{\prime}$ es la de una vocal breve; (v) en casos como el de (25c), dicho movimiento colocaría fuera de la estructura métrica la sílaba final del nexo ötı. ${ }^{9}$

Pero (25c) muestra con claridad el carácter proclítico de la negación $\mu \eta \delta \varepsilon \grave{\varepsilon}$ (cf. Vendryes 1938:67s., 251), carácter compartido por ov̉ en (25b). Por lo tanto, en estos ejemplos no existe resilabificación hacia la izquierda, análoga a la enclisis, ${ }^{10}$ sino hacia la derecha, análoga a la proclisis, como se infiere de la representación de (21). La secuencia de (25b) se analiza en la forma de (26). La palabra ortotónica está precedida por dos sílabas inacentuadas producidas por la elisión.

$$
\dot{\alpha} \lambda . \lambda^{\prime} \text { ov̉. }\langle\kappa \varepsilon \chi \omega \rho เ \sigma \mu \varepsilon \dot{v o v}\rangle
$$

De (26), se deriva entonces (27) mediante aplicaciones consecutivas de la proclisis de derecha a izquierda (se omiten los lindes de los proclíticos). 


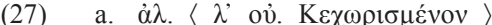

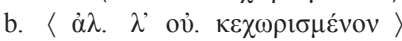

De esta manera, los segmentos desconectados adquieren visibilidad prosódica plena.

El análisis desarrollado supone que la proclisis se activa con posterioridad a la elisión, como un mecanismo al que se recurre en último término para garantizar la interpretación prosódica completa de los constituyentes, aplicándose en sentido inverso al de la enclisis (la cual procede de izquierda a derecha).

\section{Conclusión}

El proceso de elisión da respaldo independiente a la tesis de que la enclisis produce en griego antiguo palabras compuestas en la zona post-léxica de la fonología, a través del mecanismo de la adjunción. La elisión altera la estructura prosódica del input disponiendo una segmentación silábica distinta de la que proporciona el léxico. En el grupo de las palabras oxítonas que terminan en vocal breve, como secuela de la conversión del tono agudo en tono grave (esto es, la privación del acento), la elisión ocasiona el apócope de la sílaba final. Pero en todos los casos en que una palabra oxítona de tal clase va seguida de un enclítico, surge en su forma plena (sin apócope) en la superficie. Esto obedece a que la composición del ortotónico con el enclítico cancela las condiciones que permiten la elisión. En el compuesto, la última vocal del oxítono no está adyacente al borde del fin de la palabra y logra atravesar incólume los tamices de la remoción del acento y de la elisión.

\section{Notas}

1. El análisis se apoya en datos pertenecientes al ático del siglo IV. Los ejemplos se extraen primordialmente de dos obras, a saber: la República de Platón (Plat. Rep.) y la Asamblea de las mujeres de Aristófanes (Aristoph. Eccl.).

2. La forma exacta de los procesos depende de cómo se representen las vocales griegas fonológicamente.
Las reglas de (i) y (ii) ofrecen una aproximación posible:

-Redonda

-Alta

3. El dominio de la remoción es mayor que el de la elisión. Se aplica a cualquier vocal, breve o larga (incluyendo $v$ ), y a palabras que terminan en consonante.

4. La adjunción puede construirse formalmente como una especie de incorporación, en el sentido de Baker y Hale (1990).

5. Las formas que incluyen sufijos que son inherentemente tónicos no se ajustan a esta

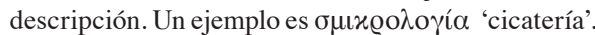

6. El acento agudo activado por la enclisis tiene función delimitadora: marca el límite final de una palabra léxicamente constituida (cf. Trubetzkoy 1939: 227, 258).

7. La composición post-léxica de (10) y (13) es una variante de la palabra de tipo E de Steriade (1988). La palabra de tipo E está constituida por una o más palabras simples de tipo W. La observación no es válida para las formas de (12), que tendrían que ser analizadas como compuestas por dos palabras de tipo E.

8. Puesto que el rasgo acentual ha sido removido, el énfasis debe expresarse en función de la estructura subyacente (adormilada) que tiene a la sílaba con acento grave como núcleo de un constituyente métrico.

9. La aféresis o prodelisión (Vendryes 1938:252, Allen 1973:227), cuando se aplica sobre la vocal acentuada, origina un compuesto análogo al de un ortotónico más un enclítico. Así, la expresión de (i), derivada por aféresis en final de verso, exhibe las propiedades prosódicas de la enclisis de (ii).

(i) $\quad \alpha$ นันố $\lambda \alpha \beta$ ov

(Aristoph. Eccl. 40) (de él tomé)

(ii) $\quad$ is $\dot{\eta} \mu \varepsilon \hat{\iota} \varsigma \phi \alpha \mu \varepsilon v \quad$ (Plat. Rep. 495b) (como decimos)

Vendryes observa que la palabra inicial de la secuencia (si es oxítona), porta acento agudo, y 
ofrece, entre otros, el siguiente ejemplo de Los Caballeros, también de final de verso, como prueba:

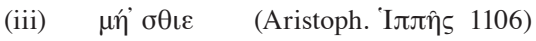

(no comer)

La edición de la obra que tengo a la vista (Atenas: Cactus), sin embargo, pone acento grave sobre la negación. El asunto requiere ulterior examen. Una cuestión que tendría que elucidarse concierne a la fase de la derivación en que se activa la aféresis.

10. La elisión determina en casos como el de (25c) la supresión del espíritu áspero (Allen 1973:229). De alguna manera, $\delta$ sustituye a la aspiración como cabeza de la primera sílaba del nexo (aunque en la escritura se mantenga por convención la marca del espíritu). Con tal análisis, se sigue que en (i), donde no hay sustitución, el segmento glotal no desaparece.

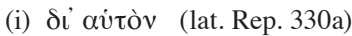

(por sí mismo)

\section{Referencias bibliográficas}

Allen, W. Sidney. 1973. Accent and rythm: Prosodic features of Latin and Greek. Cambridge: Universidad de Cambridge.

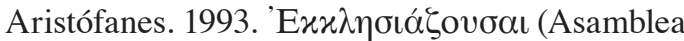
de las mujeres). Atenas: Cactus.

Aristófanes. 1994. 'I Atenas: Cactus.
Baker, Mark., Kenneth, Hale. 1990. "Relativized minimality and pronoun Incorporation". Linguistic Inquiry, 21: 289-97.

Chomsky, Noam y Morris Halle.1 968/19974. The sound pattern of English. Cambridge, Massachusetts: Imprenta del MIT.

Harris, James. 19963. "Integrity of prosodic constituents and the domain of syllabification rules in Spanish and Catalan". En: The view from building 20, pp. 177-93. Cambridge, Massachusetts: Imprenta del MIT.

Kenstowicz, Michael. 19964. "Evidence for metrical constituency". En: The view from building 20, pp. 257-73. Cambridge, Massachusetts: Imprenta del MIT.

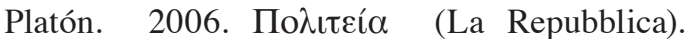
Traducción de Mario Vegetti (al italiano), con el texto griego al frente. Milán: Bur.

Steriade, Donca. 1988. "Greek accent: A case for preserving structure". Linguistic Inquiry, 19: 271-314.

Trubetzkoy, N. S. 1939/1970. Principes de phonologie. Paris: Klincksieck.

Vendryes, Joseph. 1938³. Traité d'accentuation grecque. Paris: Klincksieck. 